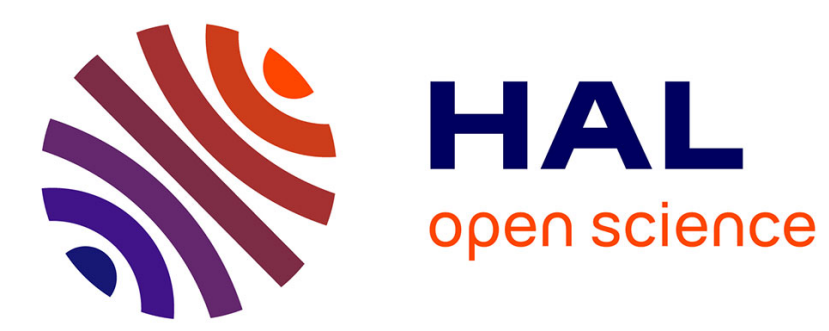

\title{
Exponential stability of a class of PDE's with dynamic boundary control.
}

Héctor Ramirez Estay, Yann Le Gorrec

\section{To cite this version:}

Héctor Ramirez Estay, Yann Le Gorrec. Exponential stability of a class of PDE's with dynamic boundary control.. American Control Conference, ACC'13., Jan 2013, United States. pp.1-6. hal00872239

\section{HAL Id: hal-00872239 \\ https://hal.science/hal-00872239}

Submitted on 11 Oct 2013

HAL is a multi-disciplinary open access archive for the deposit and dissemination of scientific research documents, whether they are published or not. The documents may come from teaching and research institutions in France or abroad, or from public or private research centers.
L'archive ouverte pluridisciplinaire $\mathbf{H A L}$, est destinée au dépôt et à la diffusion de documents scientifiques de niveau recherche, publiés ou non, émanant des établissements d'enseignement et de recherche français ou étrangers, des laboratoires publics ou privés. 


\title{
Exponential stability of a class of PDE's with dynamic boundary control
}

\author{
Hector Ramirez and Yann Le Gorrec
}

\begin{abstract}
We show that a finite dimensional strictly passive linear controller exponentially stabilizes a large class of partial differential equations which are actuated through its boundaries on a one dimensional spatial domain. This is achieved by extending existing results on exponential stability of boundary control system with static boundary control to the case with dynamic boundary control. The approach is illustrated on a physical example.
\end{abstract}

\section{INTRODUCTION}

Boundary control systems (BCS) [1] are a class of abstract systems which models partial differential equations (PDEs) with the control and the observations at the boundary of its spatial domain. A large class of physical systems may be modelled as BCS, and very powerful results on wellposedness and stability have been reported for the ones formulated using the framework of infinite dimensional portHamiltonian system [2]-[5]. More specifically in [4] it has been shown that a clever choice of the boundary conditions (static feedback) render the BCS exponentially stable, and in [3] it has been shown that for a class of BCS arising from the modelling of physical systems, a power preserving interconnection with a finite dimensional strictly positive real (SPR) [6] linear system (dynamic boundary control) results in an asymptotically stable BCS on an extended space. In this paper we extend the exponential stability result to BCS with linear dynamic boundary control. We show that if the linear finite dimensional controller is strictly passive, then the closed-loop BCS is exponentially stable on an extended space. This result permits to elegantly, and quite easily, prove the exponential stability for a large class of linear controllers, in particular those arising from energy shaping methods using Casimir functions [7], [8].

The paper is organized as follows. In Section II and III we give the preliminaries on BCS and dynamic boundary control. In Section IV we derive the main result of the paper. Section V presents a physical example and in Section VI we give some final remarks.

\section{BOUNDARY CONTROL SYSTEMS}

In the following we will briefly recall the main definitions of the BCS of interest. The reader is referred to [2], [4] and in particular to [3], [5] for further details on BCS and to

This work was supported by French ANR sponsored projects HAMECMOPSYS and Labex ACTION under reference codes ANR-11-BS03-0002 and ANR-11-LABX-01-01 respectively.

Hector Ramirez and Yann Le Gorrec are with the department of Automation and Micro-Mechatronic Systems, FEMTO-ST UMR CNRS 6174, ENSMM, 26 chemin de l'épitaphe, F-25030 Besançon, France. \{ramirez, legorrec\}efemto-st.fr
[1] for a complete exposition of infinite dimensional linear system theory.

We will follow the same notation as in [4], thus $M_{n}(\mathcal{H})$ denotes the space of square $n \times n$ matrices whose entries lie in the vector space $\mathcal{H}$. By $\langle\cdot, \cdot\rangle_{\mathbb{R}}$ we denote de inner product on $\mathbb{R}$ or $\mathbb{R}^{n}$, and $\langle\cdot, \cdot\rangle_{L_{2}}$, or simply $\langle\cdot, \cdot\rangle$ denotes the standard inner product on $L_{2}\left(a, b, \mathbb{R}^{n}\right)$. The Sobolev space of order $k$ is denoted by $H^{k}\left(a, b, \mathbb{R}^{n}\right)$. We say that a symmetric matrix is positive definite, in short $M>0$, if all its eigenvalues are positive, and positive semi-definite, in short $M \geq 0$ if its eigenvalues are non-negative. A self-adjoint operator $L$ is coercive on an inner product space $X$ if there exists an $\epsilon$ such that $L \geq \epsilon I$. The systems under study are described by the following PDE

$$
\left.\frac{\partial x}{\partial t}=P_{1} \frac{\partial}{\partial z}(\mathcal{L}(z) x)(t, z)\right)+P_{0} \mathcal{L}(z) x(t, z),
$$

$z \in(a, b)$, satisfying the following assumption.

Assumption 1. $P_{1} \in M_{n}(\mathbb{R})$ is a non-singular symmetric matrix, $P_{0}=-P_{0}^{\top} \in M_{n}(\mathbb{R})$, and $x$ takes values in $\mathbb{R}^{n}$. Furthermore, $\mathcal{L}(\cdot) \in M_{n}\left(L_{2}(a, b)\right)$ is a bounded and continuously differentiable matrix-valued function satisfying for all $z \in(a, b), \mathcal{L}(z)=\mathcal{L}(z)^{\top}$ and $\mathcal{L}(z)>m I$, with $m$ independent from $z$.

For simplicity $\mathcal{L}(z) x(t, z)$ will be denoted by $(\mathcal{L} x)(t, z)$. The state space is defined as $X=L_{2}\left(a, b ; \mathbb{R}^{n}\right)$ with inner product $\left\langle x_{1}, x_{2}\right\rangle_{\mathcal{L}}=\left\langle x_{1}, \mathcal{L} x_{2}\right\rangle$ and norm $\left\|x_{1}\right\|_{\mathcal{L}}^{2}=$ $\left\langle x_{1}, x_{1}\right\rangle_{\mathcal{L}}$. Hence $X$ is a Hilbert space. Note that the natural norm on $X$ and the $L_{2}$ norm are equivalent. The reason for selecting this space os that $\|\cdot\|^{2}$ is usually proportional to the energy function of the system.

Definition 2. [2] Let $\mathcal{L} x \in H^{1}\left(a, b ; \mathbb{R}^{n}\right)$. Then the boundary port variables associated with system (1) are the vectors $e_{\partial, \mathcal{L} x}, f_{\partial, \mathcal{L} x} \in \mathbb{R}^{n}$, defined by

$$
\left[\begin{array}{l}
f_{\partial, \mathcal{L} x} \\
e_{\partial, \mathcal{L} x}
\end{array}\right]=\frac{1}{\sqrt{2}}\left[\begin{array}{cc}
P_{1} & -P_{1} \\
I & I
\end{array}\right]\left[\begin{array}{l}
(\mathcal{L} x)(b) \\
(\mathcal{L} x)(a)
\end{array}\right] .
$$

Note that the port variables are linear combinations of the boundary variables. Let us define the matrix $\Sigma \in M_{2 n}(\mathbb{R})$ as follows

$$
\Sigma=\left[\begin{array}{ll}
0 & I \\
I & 0
\end{array}\right]
$$

Theorem 3. [2] Let $W$ be a $n \times 2 n$ real matrix. If $W$ has full rank and satisfies $W \Sigma W^{\top} \geq 0$, where $\Sigma$ is defined in (2), then the system (1), satisfying Assumption 1, with input

$$
u(t)=W\left[\begin{array}{l}
f_{\partial, \mathcal{L}_{x}}(t) \\
e_{\partial, \mathcal{L}_{x}}(t)
\end{array}\right]
$$


is a boundary control system on $X$. Furthermore, the operator $\mathcal{A} x=P_{1}(\partial / \partial z)(\mathcal{L} x)+P_{0} \mathcal{L} x$ with domain

$$
D(\mathcal{A})=\left\{\mathcal{L} x \in H^{1}\left(a, b ; \mathbb{R}^{n}\right) \mid\left[\begin{array}{c}
f_{\partial, \mathcal{L} x}(t) \\
e_{\partial, \mathcal{L} x}(t)
\end{array}\right] \in \operatorname{ker} W\right\}
$$

generates a contraction semigroup on $X$.

Let $\tilde{W}$ be a full rank matrix of size $n \times 2 n$ with $\left[\begin{array}{c}W \\ \tilde{W}\end{array}\right]$ invertible and let $P_{W, \tilde{W}}$ be given by

$P_{W, \tilde{W}}=\left(\left[\begin{array}{l}W \\ \tilde{W}\end{array}\right] \Sigma\left[\begin{array}{l}W \\ \tilde{W}\end{array}\right]^{\top}\right)^{-1}=\left[\begin{array}{cc}W \Sigma W^{\top} & W \Sigma \tilde{W}^{\top} \\ \tilde{W} \Sigma W^{\top} & \tilde{W} \Sigma \tilde{W}^{\top}\end{array}\right]^{-1}$.

Define the output of the system as the linear mapping $\mathcal{C}$ : $\mathcal{L}^{-1} H^{1}\left(a, b ; \mathbb{R}^{n}\right) \rightarrow \mathbb{R}^{n}$,

$$
y=\mathcal{C} x(t):=\tilde{W}\left[\begin{array}{l}
f_{\partial, \mathcal{L} x}(t) \\
e_{\partial, \mathcal{L} x}(t)
\end{array}\right] .
$$

Then for $u \in \mathcal{C}^{2}\left(0, \infty ; \mathbb{R}^{k}\right), \mathcal{L} x(0) \in H^{1}\left(a, b ; \mathcal{R}^{n}\right)$, and $u(0)=W\left[\begin{array}{l}f_{\partial, \mathcal{L} x}(0) \\ e_{\partial, \mathcal{L} x}(0)\end{array}\right]$ the following balance equation is satisfied:

$$
\frac{1}{2} \frac{d}{d t}\|x(t)\|_{\mathcal{L}}^{2}=\frac{1}{2}\left[u(t)^{\top} \quad y(t)^{\top}\right] P_{W, \tilde{W}}\left[\begin{array}{l}
u(t) \\
y(t)
\end{array}\right] .
$$

Remark 4. The matrix $P_{W, \tilde{W}}$ is defined if and only if $\left[\begin{array}{c}W \\ \tilde{W}\end{array}\right]$ invertible. The input and output of the system are acting on the boundary of the spatial domain, hence the system can only exchange energy with its environment through the boundaries. Finally let us do the remark that the balance equation (3) usually equals the energy of the system and that it may be rewritten as

$$
\begin{aligned}
\frac{1}{2} \frac{d}{d t}\|x(t)\|_{\mathcal{L}}^{2}=\frac{1}{2}[ & \left\langle(\mathcal{L} x)(t, b), P_{1}(\mathcal{L} x)(t, b)\right\rangle_{\mathbb{R}} \\
& \left.-\left\langle(\mathcal{L} x)(t, a), P_{1}(\mathcal{L} x)(t, a)\right\rangle_{\mathbb{R}}\right] .
\end{aligned}
$$

In [4] sufficient conditions for the exponential stability of the BCS of Theorem 3 have been given. The following Lemma is key to prove the exponential stability.

Lemma 5. [4], [5] Consider a BCS as described in Theorem 3 with $u(t)=0$, for all $t \geq 0$. Then the energy of the system $E(t)=(1 / 2)\|x(t)\|_{\mathcal{L}}^{2}$ satisfies for $\tau$ large enough

$$
\begin{aligned}
& E(\tau) \leq c(\tau) \int_{0}^{\tau}\|(\mathcal{L} x)(t, b)\|_{\mathbb{R}}^{2} d t, \quad \text { and } \\
& E(\tau) \leq c(\tau) \int_{0}^{\tau}\|(\mathcal{L} x)(t, a)\|_{\mathbb{R}}^{2} d t,
\end{aligned}
$$

where $c$ is a positive constant that only depends on $\tau$.

Using Lemma 5 it is possible to show (see [4] or [5]) that the BCS is exponentially stable if the energy of the system satisfies $(d E / d t) \leq-k\|(\mathcal{L} x)(t, b)\|_{\mathbb{R}}^{2}$ or $(d E / d t) \leq$ $-k\|(\mathcal{L} x)(t, a)\|_{\mathbb{R}}^{2}$ for some positive constant $k$. This condition is satisfied if the boundary conditions of the system are selected such that the $(2,2)$-block of the matrix $P_{W, \tilde{W}}$ is negative definite.

\section{DYNAMIC BOUNDARY CONTROL}

Dynamic control boundary implies that the BCS system is controlled through it boundaries with a dynamic control system. This implies that the boundary conditions of the infinite dimensional system are changing dynamically. In order to use some existing results from [3] we assume the following.

Assumption 6. The dynamic controller is linear, finite dimensional and strictly passive.

Let us briefly recall the concepts of dissipative, passive and positive real system for finite dimensional systems. The reader is referred to [6], [9], [10] for further details. Consider a linear system

$$
\begin{aligned}
\dot{v} & =A_{c} v+B_{c} u_{c} \\
y_{c} & =C_{c} v+D_{c} u_{c}
\end{aligned}
$$

with state space $v \in V=\mathbb{R}^{m}$, set of input values $u_{c} \in$ $U_{c}=\mathbb{R}^{n}$ and set of output values $y_{c} \in Y=\mathbb{R}^{n}$. The set $\mathcal{U}_{c}$ of admissible inputs consists of all $U_{c}$-valued piecewise continuous functions defined on $\mathbb{R} . A_{c}, B_{c}, C_{c}$ and $D_{c}$ are constant real matrices of dimension $m \times m, m \times n, n \times m$ and $n \times n$ respectively. Assume without loss of generality that the vector field $A_{c} v$ has at least one equilibrium: $A \cdot 0=0$. Let $w$ be a real valued function defined on $U \times Y$, called the supply rate. We assume that for any $u_{c} \in \mathcal{U}$ and for any $v(0) \in V$, the output $y_{c}(t)$ of (5) is such that $w(t)=w\left(u_{c}(t), y_{c}(t)\right)$ satisfies

$$
\int_{0}^{\tau}|w(t)| d t<\infty \quad \text { for all } \tau \geq 0 .
$$

Definition 7. [10] A system of the form (5) with supply rate $w$ is said to be dissipative if there exists a $C^{0}$ non-negative function $E_{c}: V \rightarrow \mathbb{R}$, called the storage function, such that for all $u_{c} \in \mathcal{U}_{c}, v(0) \in V, t \geq 0$

$$
E_{c}(\tau)-E_{c}(0) \leq \int_{0}^{\tau} w(t) d t
$$

The above inequality is called the dissipation inequality. A particularly important special class of dissipative systems are the ones with supply rate given by the inner product.

$$
w=\left\langle y_{c}, u_{c}\right\rangle=u_{c}^{\top} y_{c} .
$$

Definition 8. [10] A system of the form (5) is said to be passive if it is dissipative with supply rate $w=\left\langle u_{c}, y_{c}\right\rangle$, and the storage $E_{c}$ satisfies $E_{c}(v=0)=0$.

Hence, (5) is passive if there exists a $C^{0}$ non-negative function $E_{c}: V \rightarrow \mathbb{R}$, which satisfies $E_{c}(0)=0$, such that

$$
E_{c}(\tau)-E_{c}(0) \leq \int_{0}^{\tau} u_{c}^{\top}(t) y_{c}(t) d t
$$

Definition 9. [10] A passive system (5) with storage function $E_{c}$ is said to be strictly passive if there exists a positive definite function, called the dissipation rate, $s: V \rightarrow \mathbb{R}$ such that for all $u_{c} \in \mathcal{U}, v(0) \in V, t \geq 0$

$$
E_{c}(\tau)-E_{c}(0)=\int_{0}^{\tau} u_{c}^{\top}(t) y_{c}(t) d t-\int_{0}^{t} s(v(t)) d t .
$$


Passive systems encompass a very large class of systems, in particular port Hamiltonian systems with dissipation [6], [11], [12]. Passive systems are also positive real systems [10]. The latter can be defined as follows.

Definition 10. [10] A system (5) is said to be positive real if for all $u_{c} \in \mathcal{U}, t \geq 0$

$$
0 \leq \int_{0}^{\tau} u_{c}^{\top}(t) y_{c}(t) d t
$$

whenever $v(0)=0$.

In [3] it is shown that a power conserving interconnection [12], i.e.,

$$
\begin{aligned}
& u=r-y_{c}, \\
& y=u_{c},
\end{aligned}
$$

with $r \in \mathbb{R}^{n}$ the new input of the system, of a impedance energy preserving BCS, i.e., that satisfies $\frac{1}{2} \frac{d}{d t}\|x(t)\|_{\mathcal{L}}^{2}=$ $u(t) y(t)$, and a linear strictly positive real (SPR) finite dimensional system defines again a BCS on an extended space. SPR is a more restrictive condition than strict passivity [6]. It is possible to relax this condition on the controller and show that the power conserving interconnection with a linear strictly passive controller defines a BCS.

Theorem 11. [3] Let the state of the open-loop BCS satisfy $\frac{1}{2} \frac{d}{d t}\|x(t)\|_{\mathcal{L}}^{2}=u(t) y(t)$. Consider a LTI strictly passive finite dimensional system with storage function $E_{c}(t)=$ $\frac{1}{2}\left\langle v(t), Q_{c} v(t)\right\rangle_{\mathbb{R}^{m}}, Q_{c}=Q_{c}^{\top}>0 \in \mathbb{R}^{m} \times \mathbb{R}^{m}$. Then the feedback interconnection of the BCS and the finite dimensional system is again a BCS on the extended state space $\tilde{x} \in \tilde{X}=X \times V$ with inner product $\left\langle\tilde{x}_{1}, \tilde{x}_{2}\right\rangle_{\tilde{X}}=\left\langle x_{1}, x_{2}\right\rangle_{\mathcal{L}}+$ $\left\langle v_{1}, Q_{c} v_{2}\right\rangle_{V}$. Furthermore, the operator $\mathcal{A}_{e}$ defined by

$$
\mathcal{A}_{e} \tilde{x}=\left[\begin{array}{cc}
\mathcal{J} \mathcal{L} & 0 \\
B_{c} \mathcal{C} & A_{c}
\end{array}\right]\left[\begin{array}{l}
x \\
v
\end{array}\right]
$$

with

$$
\begin{gathered}
D\left(\mathcal{A}_{e}\right)= \\
\left\{\left[\begin{array}{l}
x \\
v
\end{array}\right] \in\left[\begin{array}{l}
X \\
V
\end{array}\right] \mid \mathcal{L} x \in H^{N}\left(a, b ; \mathbb{R}^{n}\right),\left[\begin{array}{c}
f_{\partial, \mathcal{L} x} \\
e_{\partial, \mathcal{L} x} \\
v
\end{array}\right] \in \operatorname{ker} \tilde{W}_{D}\right\},
\end{gathered}
$$

where

$$
\left.\tilde{W}_{D}=\left[\begin{array}{ll}
\left(W+D_{c} \tilde{W}\right. & C_{c}
\end{array}\right)\right]
$$

generates a contraction semigroup on $\tilde{X}$.

Proof: The proof is very similar to the one presented in [3, Theorem 5.8, pp:120]. Here we only comment on the steps of the proof and why it still holds when using a strictly passive controller instead of strictly positive real controller. The proof is performed by applying the LumerPhillips Theorem [5, Theorem 6.1.7, pp:69], which is divided in two parts: showing that $A_{e}$ is a dissipative operator (i.e. $\operatorname{Re}\langle A \tilde{x}, \tilde{x}\rangle \leq 0)$ and that $\operatorname{ran}\left(I-A_{e}\right)=\tilde{X}=X \times V$. If the controller is dissipative it is straightforward to show that $A_{e}$ is dissipative using the Kalman-Yakubovich-Popov (KYP) Lemma [6], [9]. The second part of the proof, $\operatorname{ran}\left(I-A_{e}\right)=$
$\tilde{X}$, follows if the matrix $\left(I-A_{c}\right)$ is non-singular. This is true if all the eigenvalues of the matrix $A_{c}$ are in the left half of the complex plane, which is achieved if the controller has some strict dissipation term. Booth parts of the proof hold for SPR and strictly passive systems.

Remark 12. In [3] the controller is also assumed SPR to prove the asymptotic stability of the extended system. Indeed, if the controller has a feed through term then the KYP Lemma for linear SPR system [6] is used to prove that the closed-loop system converges to a maximal invariant subset equal to $\{0\}$ and asymptotic stability follows from LaSalle's invariance principle. This is actually not necessary in the present case since LaSalle's invariance Theorem is not used to prove the exponential stability (see Section IV).

Remark 13. For Theorem 11 to be fulfilled the matrices $W$ and $\tilde{W}$ should be selected such that

$$
P_{W, \tilde{W}}=\left[\begin{array}{ll}
0 & I \\
I & 0
\end{array}\right]
$$

Notice that the power preserving interconnection (9) actually defines a feedback loop, where the finite dimensional system acts as the controller.

\section{EXPONENTIAL STABILITY}

In this section it is shown that the BCS defined in Theorem 11 is exponentially stable if the finite dimensional controller is strictly passive. For this purpose we use the same idea as in [4] and extend Lemma 5 to the present case.

Lemma 14. Consider a BCS as described in Theorem 11 with $r(t)=0$, for all $t \geq 0$. Then the energy of the system $\tilde{E}(t)=\frac{1}{2}\|x(t)\|_{\mathcal{L}}^{2}+\frac{1}{2} v(t)^{T} Q_{c} v(t)$ satisfies for $\tau$ large enough

$$
\begin{aligned}
& \tilde{E}(\tau) \leq c(\tau) \int_{0}^{\tau}\|(\mathcal{L} x)(t, b)\|_{\mathbb{R}}^{2} d t+E_{c}^{\max }, \quad \text { and } \\
& \tilde{E}(\tau) \leq c(\tau) \int_{0}^{\tau}\|(\mathcal{L} x)(t, a)\|_{\mathbb{R}}^{2} d t+E_{c}^{\max },
\end{aligned}
$$

where $c$ is a positive constant that only depends on $\tau$ and $E_{c}^{\max }$ is the maximum value of $E_{c}$ for $t \geq 0$.

Proof: In [4] it has been proved that the function

$$
F(z)=\int_{\gamma(b-z)}^{\tau-\gamma(b-z)} x^{\top}(t, z) \mathcal{L}(z) x(t, z) d t
$$

fulfils $F(b) \geq F(z) e^{-\kappa(b-a)}$ for $z \in[a, b]$ where $\kappa$ is a positive constant. On other hand due to the contraction property of the semigroup $\tilde{E}\left(t_{2}\right) \leq \tilde{E}\left(t_{1}\right)$ for $t_{2} \geq t_{1}$ it is deduced that

$$
\begin{aligned}
\int_{\gamma(b-z)}^{\tau-\gamma(b-z)} \tilde{E}(t) d t & \geq \tilde{E}(\tau-\gamma(b-a)) \int_{\gamma(b-z)}^{\tau-\gamma(b-z)} d t \\
& =(\tau-2 \gamma(b-a)) \tilde{E}(\tau-\gamma(b-a))
\end{aligned}
$$


Hence we obtain

$$
\begin{aligned}
& 2(\tau-2 \gamma(b-a)) \tilde{E}(\tau) \\
& \leq 2(\tau-2 \gamma(b-a)) \tilde{E}(\tau-\gamma(b-a)) \\
& \leq \int_{a}^{b}\left(\int_{\gamma(b-z)}^{\tau-\gamma(b-z)} x^{\top}(\mathcal{L} x) d t\right) d z+2 \int_{\gamma(b-z)}^{\tau-\gamma(b-z)} E_{c} d t \\
& \leq \int_{a}^{b} F d z+2 \int_{\gamma(b-z)}^{\tau-\gamma(b-z)} E_{c} d t \\
& \leq(b-a) F(b) e^{\kappa(b-a)}+2 \int_{\gamma(b-z)}^{\tau-\gamma(b-z)} E_{c} d t \\
& \leq c_{1} \int_{0}^{\tau}\|(\mathcal{L} x)\|_{\mathbb{R}}^{2} d t+2 \int_{\gamma(b-z)}^{\tau-\gamma(b-z)} E_{c} d t
\end{aligned}
$$

where $c_{1}=(b-a)\left\|\mathcal{L}^{-1}(b)\right\| e^{\kappa(b-a)}$. On other hand it is always true that

$$
\int_{\gamma(b-z)}^{\tau-\gamma(b-z)} E_{c} d t \leq(\tau-2 \gamma(b-a)) E_{c}^{\max ^{\prime}}
$$

where $E_{c}^{\text {max }}{ }^{\prime}$ is the maximum value of $E_{c}(t)$ for $\gamma(b-z) \leq$ $t \leq \tau-\gamma(b-z)$. Denote by $E_{c}^{\max }$ the maximum value of $E_{c}(t)$ for all $t \geq 0$, then it is true that $E_{c}^{\text {max }} \leq E_{c}^{\text {max }}$ and we obtain that for $\tau \gg 0$

$$
\tilde{E}(\tau) \leq c(\tau) \int_{0}^{\tau}\|(\mathcal{L} x)\|_{\mathbb{R}}^{2} d t+E_{c}^{\max },
$$

with $c(\tau)=\frac{c_{1}}{2(\tau-2 \gamma(b-a))}$. The second limit is obtained similarly by using the function

$$
F(z)=\int_{\gamma(a-z)}^{\tau-\gamma(a-z)} x^{\top}(t, z) \mathcal{L}(z) x(t, z) d t
$$

to develop the proof.

Theorem 15. Consider the BCS defined by Theorem 11 with $r(t)=0$, for all $t \geq 0$. If the linear finite dimensional control system is strictly passive, then the BCS system is exponentially stable.

Proof: By Theorem 11 the energy of the infinite dimensional system fulfils $(d E / d t)=u^{\top}(t) y(t)$, or in integral form

$$
E(\tau)=\int_{0}^{\tau} u^{\top}(t) y(t) d t+E(0) .
$$

The energy of the closed loop system is given by the sum of the energies of the infinite and finite dimensional subsystems and since the finite dimensional system is strictly passive it satisfies (7) and thus

$$
\begin{aligned}
\tilde{E}(\tau) & =E(\tau)+E_{c}(\tau) \\
=\int_{0}^{\tau} u^{\top}(t) y(t) d t+\int_{0}^{\tau} u_{c}^{\top}(t) y_{c}(t) & -\int_{0}^{\tau} s(t) d t \\
& +E(0)+E_{c}(0) .
\end{aligned}
$$

The subsystems are interconnected in a power conserving way, i.e., $u^{\top} y+u_{c}^{\top} y_{c}=0$, hence the total energy is given by

$$
\tilde{E}(\tau)=-\int_{0}^{\tau} s(t) d t+\tilde{E}(0)
$$

with $\tilde{E}(0)=E(0)+E_{c}(0)$. From (4) we have that

$$
\begin{aligned}
&\left|u^{\top} y\right|=\left|\left[\begin{array}{l}
(\mathcal{L} x)(t, b) \\
(\mathcal{L} x)(t, a)
\end{array}\right]^{\top} M\left[\begin{array}{l}
(\mathcal{L} x)(t, b) \\
(\mathcal{L} x)(t, a)
\end{array}\right]\right| \\
& \geq \epsilon_{1}\left\|\left[\begin{array}{l}
(\mathcal{L} x)(t, b) \\
(\mathcal{L} x)(t, a)
\end{array}\right]\right\|^{2} \geq \epsilon_{1}\|(\mathcal{L} x)(t, b)\|^{2}, \\
&\left(\geq \epsilon_{1}\|(\mathcal{L} x)(t, a)\|^{2},\right)
\end{aligned}
$$

for some $\epsilon_{1}>0$ since $M=\left[\begin{array}{cc}P_{1} & 0 \\ 0 & -P_{1}\end{array}\right]$ is symmetric and full rank [13]. From (6), (7), (8) and the definition of the dissipation rate we can always find a sufficiently small $\epsilon_{2}>0$ such that $\int_{0}^{\tau} s(t) d t \geq \epsilon_{2} \int_{0}^{\tau}\left|u_{c}^{\top} y_{c}\right| d t$. Recalling once again that $u^{\top} y+u_{c}^{\top} y_{c}=0$ and combining with (13) and (10) we have

$$
\int_{0}^{\tau} s(t) d t \geq \epsilon \int_{0}^{\tau}\|(\mathcal{L} x)(t, b)\|^{2} d t \geq \frac{\epsilon}{c(\tau)}\left(\tilde{E}(\tau)-E_{c}^{\max }\right)
$$

with $\epsilon=\epsilon_{1} \epsilon_{2}$. Inserting this expression in (12) we deduce that

$$
\tilde{E}(\tau) \leq \frac{\epsilon}{c(\tau)}\left(E_{c}^{\max }-\tilde{E}(\tau)\right)+\tilde{E}(0) .
$$

Notice that due to the contraction property of the semigroup $E_{c}^{\max }<\tilde{E}(0)$ (equality would imply $E(0)=0$ which in turn implies that the system is already at a stable equilibrium), so it is possible to write $E_{c}^{\max } \leq \epsilon_{3} \tilde{E}(0)$, for some $\epsilon_{3}<1$. Hence we finally have

$$
\tilde{E}(\tau) \leq c \tilde{E}(0)
$$

with $c=\left(\left(\frac{\epsilon_{3} \epsilon}{c(\tau)}+1\right) /\left(\frac{\epsilon}{c(\tau)}+1\right)\right)<1$. From this the semigroup generated by $\mathcal{A}_{e}$ (see Theorem 11) satisfies $\|T(\tau)\|<1$, from which exponential stability follows.

The effect of the finite dimensional strictly passive controller is the injection of damping to the global system. In [4] it has already been addressed that the exponential stability of the BCS is related to the injection of damping through the boundaries. It is interesting to do the remark that the BCS described by Theorem 11 is always exponentially stable provided that the finite dimensional subsystem is strictly passive.

\section{EXAMPLE: CONTROL OF A MICROGRIPPER}

A simplified model of a micro-gripper used for DNA manipulation [14] is presented to illustrate the previous result (cf Figure 1).

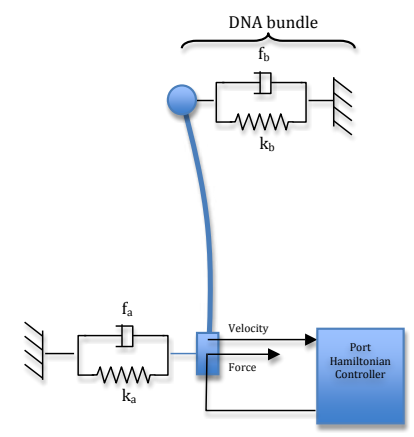

Fig. 1. DNA manipulation through port Hamiltonian control 
The trapped DNA bundle is approximated by a mass spring system attached at the tip of the tweezer. The arm is actuated by using electrostatic forces generated by a comb drive actuator attached to a suspension system. We assume that it is only possible to measure the transversal velocity at the point $a$ and act by controlling the transversal force. We also assume that the beam is clamped with respect to angular movement at the point $a$. The control strategy consists in applying a constant force $F_{a}^{*}$ to drive the system to the desired equilibrium configuration $v^{*}$. The control is completed with the transversal velocity feedback loop in order to stabilize the system around the equilibrium $v^{*}$. The problem is similar to the one solved in [7], [8] using energy shaping methods, but we will exploit the port Hamiltonian structure of the global system to define a BCS and use Theorem 15 to guarantee exponential stability. The total system, may be divided into three subsystems: The suspension mechanism at the base of the gripper, the flexible arm and the DNA-bundle at the tip of the gripper. The flexible arm is modelled as a Timoshenko beam (infinite dimensional system) while the suspension mechanism and the DNA-bundle may be modelled as finite dimensional mechanical systems.

\section{A. The Timoshenko beam}

The Timoshenko beam has been widely studied as a distributed parameter port Hamiltonian system [7] and as BCS [2] and the exponential stability of the system has been proved for static boundary feedback [3], [4]. The BCS is defined as

$$
\begin{aligned}
\frac{\partial}{\partial t}\left[\begin{array}{l}
x_{1} \\
x_{2} \\
x_{3} \\
x_{4}
\end{array}\right]=\underbrace{\left[\begin{array}{llll}
0 & 1 & 0 & 0 \\
1 & 0 & 0 & 0 \\
0 & 0 & 0 & 1 \\
0 & 0 & 1 & 0
\end{array}\right]}_{P_{1}} \frac{\partial}{\partial z}\left[\begin{array}{c}
K x_{1} \\
\frac{1}{\rho} x_{2} \\
E I x_{3} \\
\frac{1}{I_{\rho}} x_{4}
\end{array}\right] \\
+\underbrace{\left[\begin{array}{cccc}
0 & 0 & 0 & -1 \\
0 & 0 & 0 & 0 \\
0 & 0 & 0 & 0 \\
1 & 0 & 0 & 0
\end{array}\right]}_{P_{0}}\left[\begin{array}{c}
K x_{1} \\
\frac{1}{\rho} x_{2} \\
E I x_{3} \\
\frac{1}{I_{\rho}} x_{4}
\end{array}\right]
\end{aligned}
$$

where the following state (energy) variables have been defined: $x_{1}=\frac{\partial w}{\partial z}(z, t)-\phi(z, t)$ the shear displacement, $x_{2}=\rho(z) \frac{\partial w}{\partial t}(z, t)$ the transverse momentum distribution, $x_{3}=\frac{\partial \phi}{\partial z}(z, t)$ the angular displacement, and $x_{4}=I_{\rho} \frac{\partial \phi}{\partial t}(z, t)$ the angular momentum distribution, for $z \in(a, b), t \geq 0$, where $w(t, z)$ is the transverse displacement of the beam and $\phi(t, z)$ is the rotation angle of a filament of the beam. The coefficients $\rho(z), I_{\rho}(z), E(z), I(z)$ and $K(z)$ are the mass per unit length, the rotary moment of inertia of a cross section, Youngs modulus of elasticity, the moment of inertia of a cross section, and the shear modulus respectively. The matrices $P_{1}$ and $P_{0}$ defines the skew-symmetric differential operator of order 1 acting on the state space $X=L_{2}\left(a, b, \mathbb{R}^{4}\right), \mathcal{J}=P_{1} \frac{\partial}{\partial z}+P_{0}$. The energy of the beam is expressed in terms of the energy variables,

$$
\begin{aligned}
E & =\frac{1}{2} \int_{a}^{b}\left(K x_{1}^{2}+\frac{1}{\rho} x_{2}^{2}+E I x_{3}^{2}+\frac{1}{I_{\rho}} x_{4}^{2}\right) d z \\
& =\frac{1}{2} \int_{a}^{b} x(z)^{\top}(\mathcal{L} x)(z) d z=\frac{1}{2}\|x\|_{\mathcal{L}}^{2}
\end{aligned}
$$

The boundary port variables are given by [2], [3]

$$
\left[\begin{array}{c}
f_{\partial, \mathcal{L} x} \\
e_{\partial, \mathcal{L} x}
\end{array}\right]=\left[\begin{array}{c}
\left(\rho^{-1} x_{2}\right)(b)-\left(\rho^{-1} x_{2}\right)(a) \\
\left(K x_{1}\right)(b)-\left(K x_{1}\right)(a) \\
\left(I_{\rho}^{-1} x_{4}\right)(b)-\left(I_{\rho}^{-1} x_{4}\right)(a) \\
\left(E I x_{3}\right)(b)-\left(E I x_{3}\right)(a) \\
\left(\rho^{-1} x_{2}\right)(b)+\left(\rho^{-1} x_{2}\right)(a) \\
\left(K x_{1}\right)(b)+\left(K x_{1}\right)(a) \\
\left(I_{\rho}^{-1} x_{4}\right)(b)+\left(I_{\rho}^{-1} x_{4}\right)(a) \\
\left(E I x_{3}\right)(b)+\left(E I x_{3}\right)(a)
\end{array}\right] .
$$

The control objective is to control the translational and angular velocity of the DNA-bundle. However the physical ports are given by the translational force acting at the base of the beam (input), and the translational velocity at the base of the beam (output). All physical ports are hence located on the point $a$ of the beam and directly associated with the dynamic of the suspension mechanism and/or base of the beam. In order to achieve that the input and output variables of the flexible arm coincide with the physical ones we define the following input and outputs for the beam:

$$
\begin{aligned}
u & =\left[\begin{array}{llll}
v(b) & \omega(b) & -v(a) & -\omega(a)
\end{array}\right], \\
y & =\left[\begin{array}{llll}
F(b) & T(b) & F(a) & T(a)
\end{array}\right],
\end{aligned}
$$

which is achieved by defining

$$
\begin{aligned}
W & =\left[\begin{array}{cccccccc}
1 & 0 & 0 & 0 & 0 & 1 & 0 & 0 \\
0 & 0 & 1 & 0 & 0 & 0 & 0 & 1 \\
1 & 0 & 0 & 0 & 0 & -1 & 0 & 0 \\
0 & 0 & 1 & 0 & 0 & 0 & 0 & -1
\end{array}\right], \\
\tilde{W} & =\left[\begin{array}{cccccccc}
0 & 1 & 0 & 0 & 1 & 0 & 0 & 0 \\
0 & 0 & 0 & 1 & 0 & 0 & 1 & 0 \\
0 & -1 & 0 & 0 & 1 & 0 & 0 & 0 \\
0 & 0 & 0 & -1 & 0 & 0 & 1 & 0
\end{array}\right] .
\end{aligned}
$$

Notice that with this choice of input and output the BCS fulfils Theorem 11 since $P_{W, \tilde{W}}=\left[\begin{array}{ll}0 & I \\ I & 0\end{array}\right]$.

\section{B. Suspension mechanism and DNA-bundle}

The suspension mechanism and the DNA-bundle may be modelled as ideal mass-spring-damper systems, and thus both admit similar PHS representations. Let us denote by the sub-index $i=a$ the system representing the suspension mechanism and by the sub-index $i=b$ the system representing the DNA-bundle. Then we may write the following PHS

$$
\begin{aligned}
& \dot{v}_{i}=\left(J_{i}-R_{i}\right) \frac{d E_{i}}{d v_{i}}+g_{i} u_{i} \\
& y_{i}=g_{i}^{\top} \frac{d E_{i}}{d v_{i}}, \quad i=a, b
\end{aligned}
$$

where $v_{i}=\left[q_{i_{1}}, q_{i_{2}}, p_{i_{1}}, p_{i_{2}}\right]^{\top}, q_{i_{1}}, q_{i_{2}}$ are the generalized coordinates, with $q_{i_{1}}$ the distance from the equilibrium 
configuration and $q_{i_{2}}$ the rotation angle, $p_{i_{1}}, p_{i_{2}}$ are the transversal and rotational generalized momenta respectively, $J_{i}=-J_{i}^{\top}, R_{i}=R_{i}^{\top}>0 \in \mathbb{R}^{4} \times \mathbb{R}^{4}$, the interconnection and damping matrices respectively, defined as

$$
J_{i}=\left[\begin{array}{cc}
0 & I \\
-I & 0
\end{array}\right], \quad R_{i}=\left[\begin{array}{cc}
0 & 0 \\
0 & C_{i}
\end{array}\right],
$$

with $C_{i}=\left[\begin{array}{cc}c_{i_{1}} & 0 \\ 0 & c_{i_{2}}\end{array}\right] \in \mathbb{R}^{2} \times \mathbb{R}^{2}$, where $c_{i_{1}}, c_{i_{2}} \in \mathbb{R}$ are the scalar damping coefficients corresponding to the transversal and rotational translation respectively. The Hamiltonian of the system is given by the kinetic and elastic energy:

$$
E_{i}=\frac{1}{2}\left(k_{i_{1}} q_{i_{1}}^{2}+k_{i_{2}} q_{i_{2}}^{2}\right)+\frac{1}{2}\left(\frac{p_{i_{1}}^{2}}{m_{i}}+\frac{p_{a_{2}}^{2}}{m_{I_{i}}}\right)
$$

where $k_{i_{1}}, k_{i_{2}}$ are the translational and rotational spring coefficients respectively and $m_{i}, m_{I_{i}}$ are the mass and moment of inertia respectively. The total force acting on the suspension mechanism, respectively the DNA bundle, is completed with the contribution of the transversal and angular force on the point $a$, respectively point $b$, of the beam. Hence the input maps are $g_{i} \in \mathbb{R}^{4} \times \mathbb{R}^{2}, g_{i}=\left[\begin{array}{ll}0 & I\end{array}\right]^{\top}$ and the inputs $u_{i}=\left[u_{i_{1}}, u_{i_{2}}\right]^{\top} \in \mathbb{R}^{2}$ may be identified with the boundary variables of the beam at the point $a$, respectively $b$

$$
u_{i}=\left[\begin{array}{l}
u_{i_{1}} \\
u_{i_{2}}
\end{array}\right]=\left[\begin{array}{l}
\frac{\partial E}{\partial x_{2}}(i) \\
\frac{\partial E}{\partial x_{4}}(i)
\end{array}\right]=\left[\begin{array}{l}
F(i) \\
T(i)
\end{array}\right] .
$$

The outputs correspond to the transversal and angular velocity of the mass at the point $a$, respectively the point $b$, and as it has seen they correspond to the inputs at the point $a$, respectively point $b$, for the flexible arm.

The complete finite dimensional PHS may hence be written by combining the PHS representing the suspension mechanism and DNA-bundle,

$$
\begin{aligned}
\dot{v} & =\left[\begin{array}{cc|ccc}
0 & I & & \\
-I & -C_{b} & & \\
\hline & 0 & 0 & I \\
& -I & -C_{a}
\end{array}\right] \frac{d E_{c}}{d v}+\left[\begin{array}{cc}
0 & 0 \\
I & 0 \\
0 & 0 \\
0 & I
\end{array}\right] u_{c} \\
y_{c} & =\left[\begin{array}{llll}
0 & I & 0 & 0 \\
0 & 0 & 0 & I
\end{array}\right] \frac{d E_{c}}{d v}
\end{aligned}
$$

The finite dimensional PHS is a strictly passive system with state $v=\left[v_{a}, v_{b}\right]^{\top}$, Hamiltonian (storage) function $E_{c}=$ $E_{a}+E_{b}$, input $u_{c}=\left[u_{a}, u_{b}\right]^{\top}$, supply rate $w=u_{c} y_{c}$ and quadratic dissipation rate $s=\frac{d E_{a}}{d v_{a}} C_{a} \frac{d E_{a}}{d v_{a}}+{\frac{d E_{b}}{d v_{b}}}^{\top} C_{b} \frac{d E_{b}}{d v_{b}}$. Hence the microgripper i.e., the interconnection of the flexible arm, suspension mechanism and DNA-bundle is a exponentially stable system by Theorem 15 .

Let us in addition assume that we include a static feedback loop for the translational velocity of the suspension mechanism. This is equivalent to the injection of damping at the point $a$, which implies that the matrix $C_{a}$ is changed:

$$
C_{a}^{\prime}=\left[\begin{array}{cc}
\left(c_{a_{1}}+\alpha\right) & 0 \\
0 & c_{a_{2}}
\end{array}\right]
$$

where $\alpha$ is the gain of the feedback loop. Hence the static feedback loop shapes the dissipation rate $s=\frac{d E_{a}}{d v_{a}} C_{a}^{\prime} \frac{d E_{a}}{d v_{a}}+$ $\frac{d E_{b}}{d v_{b}} C_{b} C_{\frac{d E_{b}}{d v_{b}}}$ implying specifically that we can increase the damping of the system.

In [7], [8] a port-Hamiltonian controller that exploits the Casimir functions of the system to guarantee asymptotic stability is proposed. By Theorem 15 the controller exponentially stabilizes the system if it includes some dissipation term.

\section{CONCLUSION}

Theorem 15 shows that a large class of boundary control system are exponentially stable if they are interconnected in a power preserving manner with a strictly passive finite dimensional linear controller. This result adapts the exponential stability proof of [4] for static control of BCS for the case of dynamic boundary control of BCS. The approach has been illustrated on the physical example of a partially actuated micro-gripper for DNA manipulation. Here the BCS is given by the model of a Timoshenko beam and the finite dimensional controller is given by the finite dimensional subsystems: the suspension mechanism and the DNA bundle.

Future work will deal with the experimental implementation of the present example.

\section{REFERENCES}

[1] R. Curtain and H. Zwart, An introduction to infinite-dimensional linear systems theory, ser. Texts in applied mathematics. New York, USA: Springer-Verlag, 1995.

[2] Y. Le Gorrec, H. Zwart, and B. Maschke, "Dirac structures and boundary control systems associated with skew-symmetric differential operators," SIAM Journal on Control and Optimization, vol. 44, no. 5, pp. 1864-1892, 2005.

[3] J. A. Villegas, "A port-Hamiltonian approach to distributed parameter systems," Ph.D. dissertation, Universiteit Twente, 2007.

[4] J. Villegas, H. Zwart, Y. Le Gorrec, and B. Maschke, "Exponential stability of a class of boundary control systems," IEEE Transactions on Automatic Control, vol. 54, pp. 142-147, 2009.

[5] B. Jacob and H. Zwart, Linear Port-Hamiltonian Systems on Infinitedimensional Spaces, ser. Operator Theory: Advances and Applications. Basel, Switzerland: Birkhäuser, 2012, vol. 223.

[6] B. Brogliato, R. Lozano, B. Maschke, and O. Egeland, Dissipative Systems Analysis and Control, 2nd ed., ser. Communications and Control Engineering Series. London: Springer Verlag, 2007.

[7] A. Macchelli and C. Melchiorri, "Modeling and control of the Timoshenko beam. the distributed port Hamiltonian approach," SIAM Journal on Control and Optimization, vol. 43, no. 2, pp. 743-767, 2004.

[8] A. Macchelli, "Boundary energy shaping of linear distributed portHamiltonian systems," in Proceedings of the 4th IFAC workshop on Lagrangian and Hamiltonian methods for non-linear control, Bertinoro, Italy, August 2012.

[9] J. Willems, "Dissipative dynamical systems part I: General theory," Archive for Rational Mechanics and Analysis, vol. 45, pp. 321-351, 1972.

[10] C. Byrnes, A. Isidori, and J. Willems, "Passivity, feedback equivalence, and the global stabilization of minimum phase nonlinear systems," Automatic Control, IEEE Transactions on, vol. 36, no. 11, pp. 1228 -1240 , nov 1991.

[11] B. Maschke and A. J. van der Schaft, "Port-controlled Hamiltonian systems: modelling origins and systemtheoretic properties," Proceedings of the International Symposium on Nonlinear Control Systems Design, NOLCOS'92, Bordeaux, France, June, 1992.

[12] A. J. van der Schaft, L2-Gain and Passivity Techniques in Nonlinear Control, 2nd ed. New York, USA: Springer-Verlag, 2000.

[13] C. Meyer, Matrix Analysis and Applied Linear Algebra. Philadelphia, USA: SIAM, 2000.

[14] M. Boudaoud, Y. Haddab, and Y. Le Gorrec, "Modeling and optimal force control of a nonlinear electrostatic microgripper," Mechatronics, IEEE/ASME Transactions on, vol. PP, no. 99, pp. 1 -10, 2012. 